\title{
Review of Some Existing Shariah-Compliant Cryptocurrency
}

\author{
Ahmed Aliyu ${ }^{1 *}$, Kamalrulnizam Abu Bakar ${ }^{1}$, Gen Matsuda ${ }^{2}$, \\ Tasneem S. J. Darwish', Abdul Hanan Abdullah'1, Abdul Samad Ismail', \\ Raja Zahilah Raja Mohd. Radzi ${ }^{1}$, Ahmad Fadhil Yusof', \\ Mohd Murtadha Mohamad ${ }^{1}$, Mohd Yazid Idris', Zuhaimy Ismail ${ }^{3}$, \\ Ahmad Che Yaacob ${ }^{4}$, Herman ${ }^{5}$ \\ School of Computing, Faculty of Engineering, Universiti Teknologi Malaysia, \\ 281310 Skudai Johor Bahru, Malaysia. \\ OK Blockchain Centre Sdn. Bhd. Unit B19, Level 19, Tower B, Medini 9, \\ Persiaran Medini Sentral 1, Bandar Medini Iskandar, \\ 79250 Iskandar Puteri, Johor, Malaysia. \\ Mathematical Sciences Department, Faculty of Science, Universiti Teknologi \\ Malaysia (UTM), 81310 Skudai, Johor, Malaysia. \\ Faculty of Islamic Civilization, Universiti Teknologi Malaysia (UTM), \\ 51310 Skudai, Johor, Malaysia \\ Universitas Ahmad Dahlan Indonesia, \\ Daerah Istimewa Yogyakarta 55166, Indonesia \\ ${ }^{*}$ Corresponding Author \\ ahmedaliyu8513@gmail.com
}

Received: 4 June 2020

Accepted: 19 June 2020 Online First:

\begin{abstract}
Cryptocurrency has emerged as the most promising digital asset, which serves as a medium of exchange with distributed control and highly secured financial system. This lead to an increase in stakeholders' interest in digital currency investment. The cryptocurrencies majorly involve two phases including Initial Coin Offering (ICO) and blockchain creation. These phases have been challenged with criticisms in terms of the Islamic shariah injunctions. These criticisms are based on two fundamental issues including whether cryptocurrency is backed by asset and has a regulatory
\end{abstract}

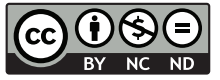


authority or not. These issues are related to uncertainty, volatility and high speculation of the cryptocurrencies. Although, several cryptocurrencies claimed to have considered the shariah requirements in their proposed cryptocurrency ecosystems. However, a strict analysis of some of the existing cryptocurrencies reveals otherwise. Therefore, this paper presents a review of some shariah-compliant cryptocurrencies focusing on their volatility and speculation. The operations of cryptocurrencies need to follow the Islamic shariah requirements. The proposed review provides an insight regarding the acclaimed shariah-compliant cryptocurrencies to assess whether they comply with the shariah requirements or not.

Keywords: Bitcoin, Cryptocurrency, Digital currency, Islamic Transaction, Shariah-compliance

\section{INTRODUCTION}

The concept of cryptocurrency emanate to limelight in the year 2008 by a renowned person from Japan called Satoshi Nakamoto (2008). Nakamoto suggests an Electronic Cash System based on Peer-to-Peer approach, which is employed for the creation of new digital currency dubbed Bitcoin (BTC or XBT). Cryptocurrency is a digital asset developed to serve as a medium of exchange that employs complex cryptography to secure operations and financial transactions. The cryptography also control the generation of additional units and validate transfer of assets. Thus, cryptocurrency is a digital currency, which serves as a substitute for fiat money. It has a limited supply, which leads to the belief that cryptocurrency can serve as money (Jenkinson, 2017). The cryptocurrency employ blockchain technology, which is based on decentralized system (Geiger, 2017). Therefore, it eliminates centralized authority from every individual and organization within the cryptocurrency ecosystem. In the blockchain, an online ledger represents the recorded transactions and the transactions are confirmed and approved using a consensus algorithms including Proof of Work (PoW), Proof of Stake (PoS), Delegated PoS (DPoS) and so on, which are carried out by digital currency miners. The blockchain system ensures that there is no repetition in the block of transaction that is, the ledger (Thompson, 2017). 
Therefore, the invention of digital currencies such as Bitcoin has enabled the creation of numerous cryptocurrencies that followed the standard of Bitcoin. Currently, there exist thousands of various cryptocurrencies and tokens, some specific differences include coins with lesser fees and more scalability for example Litecoin. Considering the expanded functions of new cryptocurrencies, discussion regarding digital currencies cannot be limited to only Bitcoin (Wood, 2014). Some of these cryptocurrencies are categorized as commodities since they are coins used as utility tokens. Thus, cryptocurrencies, for example, tokens are suitable for trade and can be used to build applications and programs onto the blockchain. Due to the creation of different nature of cryptocurrency ecosystem, there are very few discussions regarding the acceptability of cryptocurrency system in Islamic law. The use of fiat money has been generally accepted as a value for exchange of commodity and services (Adam \& Mohd Nor, 2018). The fiat money is a paper-based currency invention whereby a regulatory body called the government controls, manage its production and usage in a community (Ceruleo, 2014; Zubaidi \& Adam, 2017). Shaykh Usmani (2005) mentioned that the attributes of money with regard to the Islamic point of view include the following; i) mediun of exchange ii) unit of account and iii) store of value. Consequently, money is termed as anything that serves as a medium of exchange and is generally acceptable among people, which could be petals, paper, silver, gold and so on. Similarly, some Islamic scholars are of the view that cryptocurrency concept is permissible in Islam because it does not clearly contradict the shariah principles. Since anything can be considered as money if it satisfies the three aforementioned attributes of money thus, cryptocurrency is termed as permissible money. Aznan Hassan is of the opinion that the legality of cryptocurrency is still questionable, which requires further improvement in the Islamic jurisprudence (Aznan, 2017). Similarly, Zaharuddin mentioned that a number of Islamic guidelines must be followed in order to make buying and selling of cryptocurrency shariah-compliant (Zaharuddin, 2017). In countrary, Daud Bakar is of the opinion that cryptocurrency could be considered as halal that is, shariahcompliant since it has many attributes of the fiat money. The attributes includes value fluctuation. In addition, blockchain of cryptocurrency can be used to combat money laundry.

However, for the cryptocurrency to be fully shariah compliant, the use of the digital currency must accomplish the protection of wealth (Maqasid 
al-shariah) concept. Therefore, it is suggested that cryptocurrency should be regulated by an authority such as government and must be backed by an assets for example, oil, gold or silver in order to protect people' wealth. Furthermore, a shariah-compliant cryptocurrency is termed as a digital currency that conform with the maqasid al-shariah based on whether the cryptocurrency is been regulated by an authority and asset backed or not. Further, regarding the acceptability of cryptocurrency, the Federal Territory Mufti has stated that cryptocurrencies cannot be clearly rejected but requires more study to decide it acceptability in Islamic law. Furthermore, Pahang Mufti Datuk Seri Abdul Rahman Othman mentined that the issue of cryptocurrency acceptance based on Islamic perspective requires a comprehensive review by the expert before passing final verdict.

Islam as a religion, which provides standard guidelines for Muslims in whatever they do has made it important to checkmate even the cryptocurrency system and its operations. Al Quran has served as the main guide for Muslims, which has set rules for everything regarding human life in the world and can be applied by all mankind (Abu-Bakar, 2018). The whole aim of Islamic law is to protect and achieve benefit for humanity. In financial transactions, Islam has offered its own rule and regulations regarding Islamic financial system. The rules and regulations regarding financial systems state that there should be no activities involving gambling (maysir), interest (riba) and uncertainty (gharar). Any activity related to one or more of the three aforementioned shariah injunctions is termed as forbidden (Meera, 2018). The maysir, which is gambling is referred to as wagering of a valuable item such as money on an event with an indeterminate or uncertain outcome. The riba is factually seen as al-ziyadah, which is the addition of gain in a transaction without loss in the case of failed business. Gharar means uncertainty due to deception and unclear risk involved in a transaction (Abu-Bakar, 2018). All these three concepts of the Islamic injunctions can be associated with the existing cryptocurrencies system since they are used as a store of value and medium of exchange.

Several stakeholders have debated that digital cryptocurrencies are not real money because they are not backed by a tangible asset that has intrinsic value. Hence, for cryptocurrency to be termed as money, it must be a store of value, medium of exchange, serve as a standard of deferred payment and has a unit of account (Bank of England, 2014, in Adam, 2017). 
Consequently, the Islamic world has been holding back their interest in accepting cryptocurrency because the process does not fully abide by the Shariah injunctions regarding financial transactions (Abu-Bakar, 2018). The demand of the Islamic world cannot be ignored considering the immense growth of the global halal industry worldwide. Further, Islamic finance has reached up to one hundred and sixty-two trillion United States dollars (USD $\$ 162$ trillion) in the global commercial banking assets (BayanToken, 2018). However, investment in the cryptocurrency market has been growing very slowly in the Islamic community. This is in connection with the unclear and dubious ruling of cryptocurrency, which is not within the shariah scope.

Consequently, many cryptocurrencies have been designed to address the limitation of the Bitcoin in the aspect of the shariah principles. The existing cryptocurrencies that claimed to be shariah compliant includes OneGram, NoorCoin, Bayan Token, ADAB and Stellar cryptocurrency. However, almost all of these cryptocurrencies have not explicitly defined how they comply with the shariah injunctions. Some research works have been conducted to provide insight into the compliance of cryptocurrency with the shariah injunctions. However, there exists no research work that provides a review and comparative discussion of the existing shariah-compliant cryptocurrencies. The review is conducted from the cryptocurrency white papers and websites, due to limitation of research materials. Therefore, in this paper, comparative discussions of some current shariah-compliant cryptocurrencies have been discussed based on two metrics including backed by asset and regulatory authority. Precisely, the following contributions have been highlighted in this paper:

1. An extensive review of the existing cryptocurrencies considering both shariah compliant and non- shariah compliant cryptocurrencies.

2. A comparative discussion of the existing shariah-compliant cryptocurrencies focusing on their operations/transaction.

3. A highlight on challenges of implementing shariah-compliant cryptocurrency.

The remaining parts of the paper are structured as follows: Section 2 contains a review of related works that are based on both shariah compliant 
cryptocurrencies and non-shariah compliant cryptocurrencies. In Section 3, the foundation of cryptocurrency based on bitcoin has been discussed. In Section 4, comparative discussion of the shariah-compliant cryptocurrencies is presented. Further, Section 5 entails highlights of challenges in implementing shariah compliant cryptocurrency. Lastly, conclusion and recommendations are put forth in Section 6.

\section{RELATED WORKS}

In this section, an extensive review of most recent review works regarding the acceptability of cryptocurrency based on the shariah perspective has been presented. The general concept of money has been discussed by relating it to cryptocurrency in several research works. Money in a generic termed has been considered as anything that is commonly acceptable as a medium of exchange, which has a store of value. The money must not have a distinctive nature or form (Turki, 1988). Al-kuwaitiyah (1983) has also mentioned that money can be anything, which has a store of value and serve as medium of exchange for example, paper, flower, pertals, skin, silver, gold and so on. Further, Usmani (2010) expressed money as something that has three attributes including medium of exchange, unit of account and store of value. Meanwhile, the evaluation of cryptocurrency by some scholars based on the aforementioned attributes suggest that cryptocurrency is shariah compliant, but requires some modification in order to reduce the risk involved in terms of it operation. The related risk in the cryptocurrency includes uncertainty and volatility, which are due to lack of monitoring authority and tangible asset backing thecryptocurrency.

Meera (2018) has studied the implication of bitcoin with regard to Islamic finance and further questioned its acceptability for the medium of exchange considering its compliance with the shariah injunctions. The study is based on analysis of the bitcoin nature and its characteristics. The analysis of the study demonstrates that any cryptocurrencies, which is not backed by real and tangible assets cannot be shariah-compliant. Meanwhile, most of the shariah scholars are presently researching towards accepting the bitcoin based on maslahah. Bitcoin is neither real money nor fiat money. The lack of intrinsic value coupled with the absence of supervision by a regulatory body such as central bank might lead to exploiting the bitcoin currency. 
In addition, the study also contends that the bitcoin has the element of prohibited concept in Islamic shariah injunction, which includes gambling (maysir) and uncertainty (gharar). Thus, this can cause socio-economic unfairness, which leads to disobeying the maqasid al-shariah. The gambling is majorly an Islamic shariah-compliant issue in the transaction level of the cryptocurrency. The deduction of the study state that the cryptocurrency is not shariah compliant.

One of the most successful types of cryptocurrency in blockchain management is bitcoin, which has become the most popular in recent years. The fundamental issue regarding the cryptocurrency is its legitimacy in terms of tax evasion, money laundering, and lack of regulation by a regulatory body. Abubakar et al., (2018) explored the opinion of Muslim scholars on the legitimacy and legality of bitcoin with regard to shariah injunctions. The research study uses the doctrinal method, which employs a descriptive method of qualitative research methodology that depends on secondary data in form of a newspaper, Journals, textbooks, and related websites. The findings show that Muslim scholars do not have a unanimous agreement on the acceptability of cryptocurrency. Some of the Muslim scholars believe that bitcoin does not oppose the Islamic principles, but requires some certain amendments. While other scholars outrightly reject the bitcoin because of its contradiction with Islamic injunction. Meanwhile, most of the researchers are inclined to supporting the view that cryptocurrency only requires amendments on the policy and regulations in order to be shariah compliant, but not to be completely rejected (Abubakar et al., 2018).

There have been different views regarding the fiat money system and the digital cryptocurrencies. Some researchers have argued that it is better to have a substitute for the existing fiat money system. The predominant role of the fiat money is carried out by the banks while the cryptocurrency does not have any bank account, auditing, and tax payment. Others do not agree with these views and have claimed that any means of payment that differs from the traditional mode including cash payment and cheques will lead to avoiding auditing and tax payment. This will cause a severe challenge to government budget and a general decline in GDP. Oziev and Yandiev, (2018) employs analytical, theoretical and descriptive methods in the findings of the following points i) defining cryptocurrency in the financial system; ii) reviewing existing literature on the topic; iii) unveiling the crucial 
requirements of shariah for money circulation; iv) comparing the features of fiat money and cryptocurrencies. The study deduced that most of the authors agree to the permissibility of cryptocurrency but with amendments.

The aforementioned related works have critically reviewed the existing views of the Islamic scholars regarding the acceptability of cryptocurrency. However, extensive comparative analysis of the existing shariah-compliant cryptocurrencies such as Bayan token, OneGram, and NoorCoin have not been explored. Hence, this paper explores the most recent shariah compliant cryptocurrencies.

\section{BITCOIN CRYPTOCURRENCY}

In this section, the general concept of Bitcoin (BTC) is first discussed, which serve as a foundation for the comparative discussion of the most recent shariah-compliant cryptocurrencies. The initial introduction of BTC is essential because it has become the basis for most of the existing cryptocurrencies. BTC is an electronic payment system that uses cryptography for its anonymity with non-centralized virtual currency, which is based on the peer-to-peer distribution of currency (Nakamoto, 2008). The bitcoin does not have any financial organization that is managing it. The significant concept of bitcoin cryptocurrency includes initial coin offering, digital wallet, transactions, the consensus system (proof of work) and mining see Diagram 1.

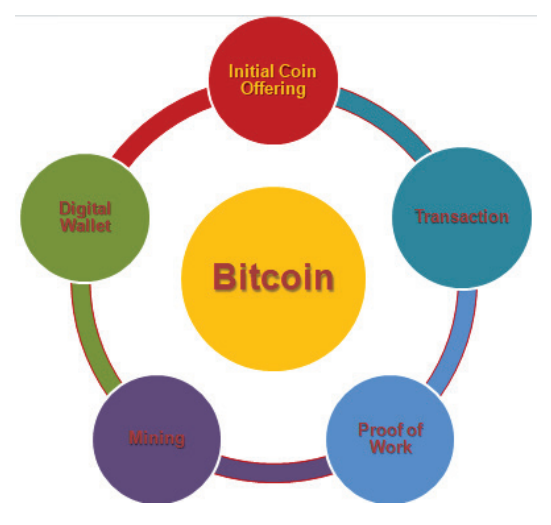

Diagram 1: Bitcoin Key Concepts 
At the initial stage of creating the BTC cryptocurrency, the Initial Coin Offering (ICO) is conducted. The ICO is a new process of funding a project for start-ups in which new digital coins or tokens are issued, is also called tokenization. Firstly, a start-up can generate a new digital token or cryptocurrency through different platforms. Then the cryptocurrency company will perform public ICO so that retail investors can purchase the new digital token. Every investor pays for the coin with the native currency of the network platform. However, in the existing ICO process, only Know Your Customer (KYC) is considered in the case of money laundry and drug trafficking in order to avoid criminal deals or prohibited transaction. Typically, each investor has to provide personal details in order to purchase bitcoins. The common bitcoin exchange Mt. Gox for instance just lately augmented its KYC requirements. Any investor who wants to deposit or withdraw coins other than BTC has to offer a scan of his national ID (Mt Gox, 2013). To unlink the BTC from An investors' real identity, a mixing service to transfer BTC to a new and anonymous address is employed. BTC mixes are services, which claim to improve anonymity by mixing the currencies of multiple investors, making it difficult to find a relation amid input and output transactions in the transaction chart. Meanwhile, companies that are into the sales of the non-halal product are not carefully avoided in the public ICO.

The BTC transactions are direct between the sender and receiver by the means of broadcasting via a peer-to-peer network. The BTC transaction is anonymous and is made public to every node within the network. An initiated transaction remains unauthorized until Proof of Work (PoW) consensus system is executed. Therefore, the PoW is employed as a mechanism for approving transactions considering the reward system and security. It is a mechanism that challenges the mining nodes in terms of high puzzle computation for transaction validation. The approval of the transaction leads to the creation of bitcoin, which is saved in the digital wallet and transaction blocks are updated (Gobel et al., 2016). The nodes in the network perform the collection of the transaction. The collections of the interrelated blocks of the transaction are called Blockchain. The transaction validation involves mining process because there is no central system for establishing trust in order to generate the BTC. The mining process computes the PoW consensus from a transaction block. This process enhances the security while at the same time approves the transaction. Thus, for every transaction approved 
by the user (miner) a reward is given. In the BTC cryptocurrency, the larger the number of miners the higher the security of the network (Aitzhan and Svetinovic, 2018). However, the BTC PoW transaction is very slow, as it requires about 10 seconds for performing a single transaction. Another issue is the rewarding system of the PoW, which allocate reward that is not commensurate with the effort of the miner. Even though some authors have claimed that the miners' machine is a high computational device that consumes more electricity, therefore, the electricity consumed must be paid for. Thus, they conclude that reward needs to be given tominers.

The purchased or generated BTC is stored in a digital wallet, which can be in form of local applications or web services. In the digital wallet, the BTC is secured using private and/or public keys. Digital currency in the wallet cannot be freeze like in the case of a conventional bank account. However, if the private key is a loss, then the BTC is gone forever from the BTC economy. Thus, BTC has some challenges including not being a shariahcompliant cryptocurrency. Further, some shariah compliant cryptocurrencies including OneGram, NoorCoin and Bayan token cryptocurrencies are analyzed and discussed in the following subsections.

\section{REVIEW OF SHARIAH-COMPLIANT CRYPTOCURRENCY}

In this section, a review and comparative discussions of the existing cryptocurrencies have been presented focusing on whether it is shariahcompliant or not considering the two metrics including regulatory authority and assetbacked.

\section{OneGram Cryptocurrency}

The OneGram cryptocurrency is a digital currency that is backed by a gold asset, where each gram of gold represents one coin. It development start in May 2017 and is domiciled in Dubai. Considering the stability of OneGram asset and commodities, about 1.8 billion people have considered using the business platform of the gold coin. Thus, it has entered sevel countries including Malaysia. The spots price of the digital currency depends on the current market value of gold. Thus, the usage and market 
demand adds a premium to the value of the OneGram currency. Therefore, the OneGram has three-valuation component to ascertain its total market price. The first portion is the Gold Value (GV), which is determined by the spot price of gold. The second portion is the current value of the Transaction Fees (TF) reinvested to purchase more gold, which the value is determined by the level of utilization of the OneGram coin. Then the third portion is the Economic Value (EV) that is being determined by the market demand. Hence, the following formula in Eq. 1 is employed for ascertaining the market price.

$$
\text { OneGram coin }=G V+E V
$$

Considering the transaction aspect of the OneGram, each transaction creates $1 \%$ transaction fee, up to a maximum of OneGram token. The OneGram is different from other cryptocurrencies because $70 \%$ of the generated coins are reinvested to purchase more gold to grow the quantity of gold that backs each token. Thus, as transaction volumes upsurge, then more gold is deposited to the vault. All OneGram investors share from the profit due to uplift in the value of each unit. As such, after some time, the value of each OneGram token rises naturally. This makes OneGram a distinctive cryptocurrency and a reliable store of wealth whose worth increases continuously. Further, development and operation of the OneGram investment are funded using $25 \%$ of the fees generated. Meanwhile, $2.5 \%$ is assigned to charity donation and another $2.5 \%$ is allocated for miners rewards. In order for OneGram to have liquidity and rapid real-world adoption, some strategies including; i) Gold guard platform for redeeming gold in relation to the issued OneGram coins is provided ii) a novel payment gateway called YalaPay, is launched for OneGram coins iii) a Gold guard MasterCard debit card (Liquid Gold) for OneGram liquidity is created (Khan,2017).

However, the OneGram ecosystem employs the KYC concept in order to have background information of its investors to deal with bad activities. It also has the gold guard company, which handles the gold storage and an Accounting and Auditing Organization for Islamic Financial Institutions (AAOIFI), which provides the rules guiding the cryptocurrency. Further, as mentioned earlier, the cryptocurrency is backed by gold. Thus, the issue of uncertainty, speculation and volatility of the non-shariah-cryptocurrency 
Journal of Contemporary Islamic Studies

has been addressed. Therefore, the OneGram can be considered as a shariah compliant cryptocurrency in terms of uncertainty and volatility.

\section{NoorCoin Cryptocurrency}

The NoorCoin is designed based on smart contract technologies ERC20 standard. It is the first shariah compliant token in the world, which is first based in Jakarta Indonesia. Application demo of NoorCoin token has been launched in 57 Organization of Islamic Cooperation (OIC) member countries. As stated by the CEO and founder of NorCoin (Sofia Koswara), the kingdom of Saudi Arabia is very keen on using the NoorCoin. The financial institutions of the kingdom have recommended the token to the Zakat Council for possible consideration. NoorCoin is another form of cryptocurrency, which has been awarded a shariah certificate from an organization called world Sharia Advisory Committee. The NoorCoin cryptocurrency employs a zilliqa platform. The platform is a highperformance public blockchain developed to scale thousands of transactions per second. The consensus system employed in zilliqa is practical Byzantine Fault Tolerance (pBFT). The pBFT has some advantages including low intensive computation, leverage a lesser consensus set efficiency and provides finality to transactions. The present transaction proficiency of zalliqa is up to 2500 transactions/second, which is in dissimilarity to BTC and Ethereum, which can only support 3-15transaction/second.

The transaction aspect of the NoorCoin charge $1.5 \%$ transaction fee from investors, which $0.5 \%$ of the $1.5 \%$ is allocated to the voucher smart contract for the coin token investor to use it in the ecosystem. While $1 \%$ is assigned to the NoorCoin body as revenue. It is shariah compliant based on these conditions including; i) there is no usury (interest) in the issuance of NoorCoin ii) loss and profit are shared as part of rewarding mechanism iii) there is lesser speculation because the cryptocurrency is backed by an asset (Noorcoin, 2018). However, the asset is not mentioned in the whitepaper.

However, the NoorCoin also employs the KYC strategy in order to have the background information of its investors so as to avoid unclean activities in the cryptocurrency platform. It also has the GoldGuard company, which handles the gold storage and other gold exchange matters. Though, as mentioned earlier, the cryptocurrency is not clearly backed by a physical 
asset. Thus, the issue of uncertainty, speculation and volatility might be related to the NoorCoin. Therefore, NoorCoin can not be clearly considered as a shariah compliant cryptocurrency in terms of uncertainty and volatility.

\section{Bayan Token Cryptocurrency}

Bayan token is an ERC20 token that is built on the Ethereum platform. It is a cryptocurrency token, which is based on utility and is also backed by tangible asset in the form of the private cloud server. Its growth is continued by economic projects, which have an influence on the society. Each Bayan token contract denotes 16 hours of access benefits of the private cloud server. Each cloud server has a configuration of 1GB memory, 5GB SSD disk, $2 \mathrm{GHz}$ and $10 \mathrm{~GB}$ bandwidth per month of transfer. Thus, token investors can redeem usage of the cloud server through smartcontract.

The Bayan token is shariah compliant token considering these attributes; i) technology is centered on token and projects ii) token has intrinsic value and real economic benefit iii) token with form of offering and potential return iv) tokens are aligned with Environmental, Social and Governance (ESG) v) token has Social Responsible Investment (SRI) standard and great utility features hence, ensuring sustainability and growth of the token. However, the data, which is based on a cloud server, is only usable in countries under the cloud computing service region. In addition, only token with the same backed value is tradable. To handle the issue of money laundering activities and terrorist financing due to abuse of cryptocurrency, a KYC strategy is introduced for each token owner. In the Bayan token, the KYC link is provided on the dashboard of the platform. In order to make Bayan token to have liquidity and be circulated in the market, Bayan token will be made usable for buying goods at some specific registered shop, for example, Elzar Mart and other online and offline marts. Each investor is considered as a holder of exclusive virtual membership card such that every investor has a specific privilege and entitlements in the future, for example, discounted price when buying goods at certain stores or restaurant. The gain from the Bayan token ICO will be used to fund projects that will guarantee the upsurge of Bayan token as a valuable token (BayanToken, 2018). 
Meanwhile, the Bayan token also consider the know your customer strategy in order to combat money laundry and other unclean activities by investors. As mentioned earlier the token is backed by data (cloud server). However, regulating authority for Bayan token is not clearly stated. Thus, the issue of uncertainty might arise since there is no clear regulatory authority for the token operation. Therefore, Bayan token can be considered as a shariah compliant cryptocurrency in terms of uncertainty, but might have some element of risk since there is no regulatory body.

\section{ADAB Solution}

The ADAB solution concepts proposed to enact a shariah advisory board within a sustainable platform for starting the halal project. The creation of ADAB solution will allow the formation of an interested community that will formalize the work of cryptology based on shariah rules. The proposed shariah advisory board will conduct an extensive and systematic study of the cryptocurrency market and its elements for compliance with the shariah injunctions. Based on the ADAB concept of the cryptocurrency, the price of a token rise as the quantity of the token decreases. Further, ADAB solution has a charity foundation that uses $2.5 \%$ of the quarterly $5 \%$ net income of the annual net profit of the project, which will form the source of the charity fund (ADAB solution, 2017). It also state that non-shariah compliant project activities including immoral services, financial pyramid and production of unlawful product such as alcoholic beverages and tobacco will not be approved.

Meanwhile, the ADAB solution also employs the KYC concept for combating money laundry and other unclean activities by investors. However, it is not backed by any tangible assets and also, a regulatory body, which oversee the ADAB token solution is not mentioned in the white paper. Thus, the issue of uncertainty, volatility and speculations might occur since there is no clear regulatory authority and not backed by tangible asset. Therefore, ADAB token can not be clearly considered as a shariah compliant cryptocurrency in terms of volatility, speculation and uncertainty. 


\section{Stellar Cryptocurrency}

The stellar cryptocurrency is a Californian based cryptocurrency that has obtained certification from Islamic scholars for its blockchain ecosystem and the coin itself, with the target to integrate the technology into financial products that are shariah-compliant. The shariah certification and the guidelines for kinds of assets to be traded on the Stellar platform are offered by the Shariyah Review Bureau (SRB), which is an Islamic advisory organization that is licensed by Bahrain central bank (Mazieres, 2016). The native currency of the stellar is called Lumen, which is almost the $7^{\text {th }}$ cryptocurrency with a market cap of $\$ 4.3$ billion. However, the main issue to be considered is the use of underlying cryptocurrency said by Mansoor Ahmed, the assistant manager at SRB.

However, the Lumen is not also backed by any tangible assets, which is an essential factor for any cryptocurrency to be shariah compliant. Meanwhile, the ADAB also employs the KYC concept for addressing the issue of bad activities by investors. However, there is no regulatory body, which oversea the lumen cryptocurrency activities mentioned in the white paper. Thus, the issues relating to uncertainty, volatility and speculations could arise since there is no clear regulatory authority and not backed by tangible asset. Therefore, stellar cryptocurrency can not be clearly considered as a shariah compliant cryptocurrency in terms of volatility, speculation and uncertainty.

Table 1 represents the summary of the shariah compliant cryptocurrencies considering the shariah requirements including unlawful industries, uncertainty (gharar), and asset-backed. From the comparison table considering KYC for unlawful industry and investors, backed by asset and regulatory body. Thus, OneGram can be considered as shariahcompliant. The NoorCoin might not be considered as shariah-compliant since is not backed by a tangible asset. Bayan token might be considered as shariah-compliant because it is backed by a tangible asset. The ADAB cryptocurrency solution might not be considered as shariah-compliant because is not backed by a tangible asset. Last, the Stellar is also considered as non-shariah compliant cryptocurrency because it is also not backed by asset. 
Journal of Contemporary Islamic Studies

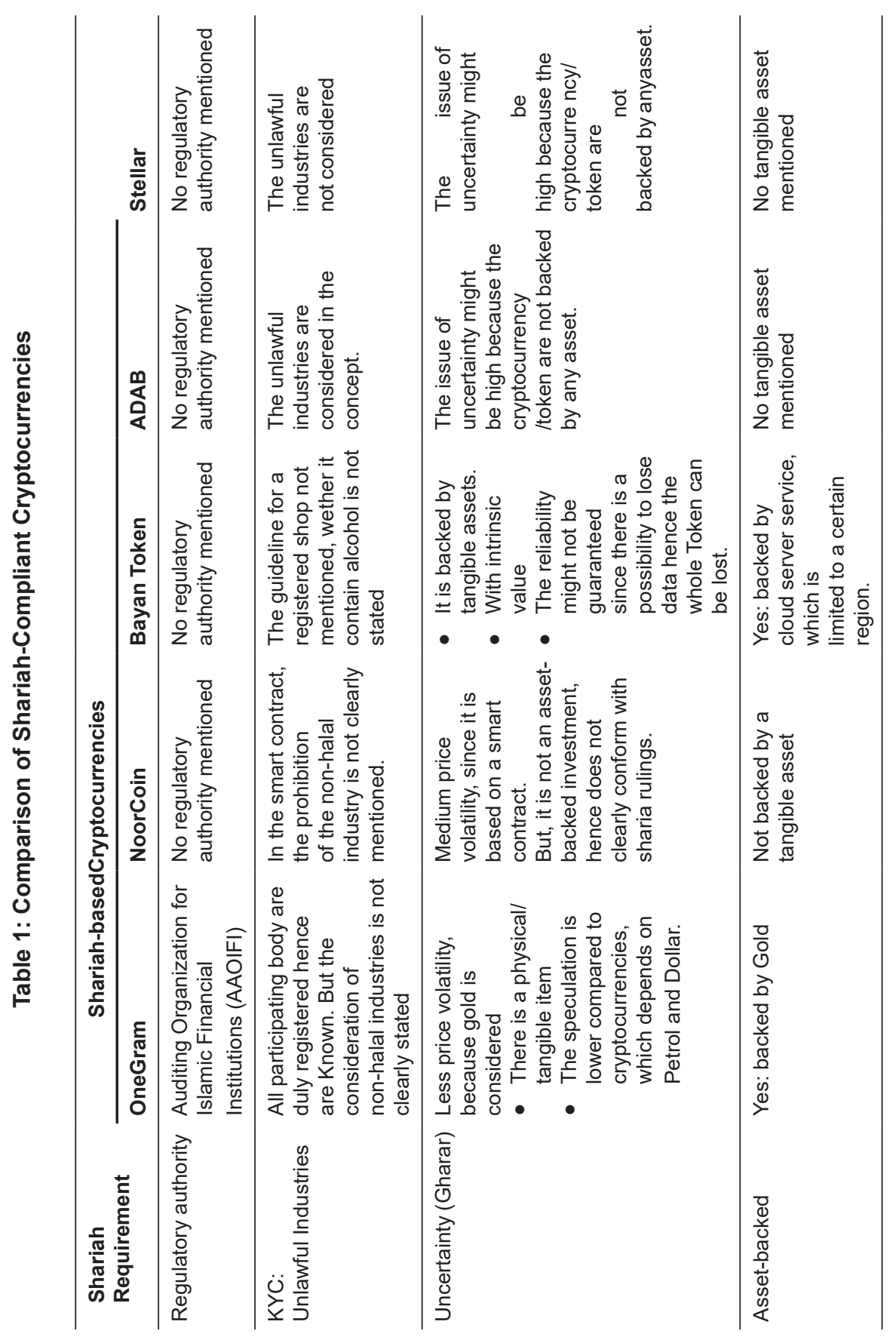




\section{CHALLENGES OF SHARIAH COMPLIANT CRYPTOCURRENCY}

Some related challenges have been presented in this section considering shariah-compliant cryptocurrencies. Several companies have attempted to back cryptocurrencies with specific assets, for example, gold by Khan et al. (2017). However, the asset-backed cryptocurrency will only turn into a marketing mechanism for enticing investments assured by real collateral, but will no longer be a cryptocurrency anymore because it requires a central regulatory body. In the Islamic rule, there is a need for a monitoring organization to regulate the operation of the cryptocurrency as stated by Abu Bakar (2018). Meanwhile, this concept might jeopardize the effort of the decentralization system of cryptocurrency. Another issue with the cryptocurrency is the issue of tax evasion, in the cryptocurrency, every user and transaction are anonymous in carrying out operations. Thus, this can make companies and users avoid tax payment or even not reporting to the tax authorities. Consequently, the country stands the risk of losing some of its tax revenue (Marian, 2013). Further, there is a challenge regarding lack of transaction recovery of digital currency, in a situation whereby a transaction is performed wrongly, by interchanging the field for commission for payment with a field for sending amount. Then the money transferred is loss and cannot be reversed, contrary to the case of bank regulatory body (central bank), which can recover/reverse transaction. In addition, if the digital wallet login detail such as a password is lost, then no one can get access to the digital currency (money) (Marian, 2013). However, all these challenges are not only related to shariah-compliant cryptocurrency but also to non-shariah-compliant cryptocurrency. Therefore, there is a need to look into these issues.

\section{CONCLUSION}

In this paper, an extensive review of the related works on shariah compliant cryptocurrencies have been conducted based on the most recent developments. The conformity of cryptocurrencies with the Islamic shariah law has become one of the major issues in the Islamic financial system in the Muslim society. The compliance of cryptocurrencies with the shariah law will encourage the acceptance of cryptocurrency. It has 
been revealed that about $\$ 162$ trillion are generated from Islamic finance transactions throughout the Islamic countries. Therefore, the acceptance of cryptocurrency in the Islamic society will go a long way in enhancing businesses globally. Several cryptocurrencies have been developed in order to address the issue of non-compliance of cryptocurrencies with the Shariah law. However, these cryptocurrencies are not fully compliant with the shariah considering the different verdict of the Islamic scholars regarding the digital currency. Thus, this paper presents discussions regarding the existing acclaimed shariah compliant cryptocurrencies and further, highlights some challenges of shariah compliant cryptocurrencies.

\section{ACKNOWLEDGEMENTS}

This research is funded by OK Blockchain Center Sdn. Bhd. and OKWAVE Co. Limited, and conducted in collaboration with University Teknologi Malaysia (UTM). UTM Research Management Centre VOT NUMBER is $4 \mathrm{C} 209$.

\section{REFERENCES}

Abdullah, A., \& Rizal, M. N. (2018). A Framework for the Development of a National Crypto-Currency. International Journal of Economics and Finance, 10, 14-25 (2018). doi:10.5539/ijef.v10n9p14.

Abubakar, Y. S., Ogunbado, A. F. and Saidi, M. A. (2018). Bitcoin and its Legality from Shari'ah Point of View. SEISENSE Journal of Management, 1, 13-21 (2018). DOI: https://doi.org/10.5281/ zenodo.1400535, 13-21.

Abu-Bakar, M.M., (2018). Shari'ah Analysis of Bitcoin, Cryptocurrency, and Blockchain: http://www.istisna.kz/eng/img/Shariah-Analysis-ofBitcoin-Cryptocurrency Blockchain. pdf. Accessed 20 Nov 2018.

ADAB Solutions (2017). ADAB cryptocurrency white paper. https:// neironix.io/documents/whitepaper/6187/WhitePaper_of_ADAB_ Solutions.pdf. Accessed 2 Nov 2018. 
Adam, M. F. (2017). Bitcoin: Shariah Compliant? Amanah Finance Consultancy,. Available Online:http://darulfiqh.com/wp-content/ uploads/2017/08/ResearchPaper-on-Bitcoin-Mufti-FarazAdam.pdf. Accessed 12 Sept 2018.

Aznan H. (2017) Shariah and Fintech Solution in Wealth Management, Institute of Islamic Banking and Finance, International Islamic University Malaysia.

Aitzhan, N.Z. and Svetinovic, D. (2018). Security and privacy in decentralized energy trading through multi-signatures, blockchain and anonymous messaging streams. IEEE Transactions on Dependable and Secure Computing, 15, 840-852 (2018). doi: 10.1109/TDSC.2016.2616861.

Al-Kuwaitiyah, A., A. (1983). Wizarat al-Auqaf wa al-Syu'un al-Islamiyah, Kuwait. Bank of England. (2014). 2014 Q1. Quarterly Bulletin, 54, 1.

Bayan Token. (2018). A Shari'ah-compliant utility crypto token white paper (V3.9). https://bayantoken.com/files/frontend/BayanatToken Whitepaper-v3.9.pdf. Accessed 17 Nov 2018.

Ceruleo, P. (2014). Bitcoin: a rival to fiat money or a speculative financial asset?. https://tesi.luiss.it/13806/1/ceruleo-paola-tesi-2014.pdf. Accessed 2 Sept 2018.

Daud Bakar, M. (2018). Is Cryptocurency Haram? Accessed 26 June 2020. www.vulcanpost.com.

Geiger, P. (2017). Get into Bitcoin before the next financial crisis. Medium. https://medium.com/@philgeiger/get-into-bitcoin-before-the nextfinancial-crisis-f0707e2d56c1. Accessed 11 Sept2017.

Göbel, J., Keeler, H. P., Krzesinski, A. E. \& Taylor, P. G., (2016). Bitcoin blockchain dynamics: The selfish-mine strategy in the presence of propagation delay. Performance Evaluation, 104, 23-41 (2016). doi: arXiv:1505.05343v2. 
https://www.hmetro.com.my/mutakhir/2018/01/300371/jawatankuasafatwa-dapatkan-pendapat-pakar-berkaitan-bitcoin. Accessed 10 Jan 2019 http://www.muftiwp.gov.my/ms/artikel/bayan-linnas/2773-bayanlinnas-153-hukum- penggunaan-mata-wang-bitcoin. Accessed 10 Jan 2019.

Jenkinson, G. (2017). Keiser-Bitcoin like 'Moses’ for Gold. Coin Telegraph. December 5, 2017. Available Online: https://cointelegraph.com/news/ keiser-bitcoin-like-moses-forgold.

Khan, I., M. (2017). Read the OneGram white paper. https://icobazaar. com/storage/campaigns/1109/whitepaper.pdf. Accessed 13 Nov2018.

Marian, O., Y. (2013). Are Cryptocurrencies Super'Tax Havens? https:// papers.ssrn.com/sol3/papers.cfm?abstract_id $=2305863$. Accessed 14 Sept2018.

Mazieres, D. (2016). The stellar consensus protocol: A federated model for Internet-level consensus. Stellar Development Foundation white paper. Accessed 2 Dec 2018. https://whitepaperdatabase.com/stellarlumens-xlm-whitepaper/.

Meera, A., K., M. (2018). Cryptocurrencies from Islamic Perspectives: The Case of Bitcoin.

Bulletin Ekonomi Moneter Dan Perbankan, 20, 475-492 (2018).

Gox, Mt. (2013). Statement regarding Account Verifications. https://mtgox. com/press_release_20130530.html. Accessed 23 October 2018.

Muedini, F. (2018). The Compatibility of Cryptocurrencies and Islamic Finance. European Journal of Islamic Finance, 10, 1-10, (2018). doi: http://dx.doi.org/10.13135/2421-2172/2569.

Nakamoto, S. (2008). Bitcoin: A Peer-to-Peer Electronic Cash System. https://bitcoin.org/bitcoin.pdf. Accessed 2 Oct 2018. 
Noorcoin, (2018). Noorcoin white paper. https://www.noorcoin.io/ NoorcoinWhitepaper.pdf. Accessed 15 Nov 2018.

Oziev, G., \& Yandiev, M. (2017). Cryptocurrency from Shari'ah Perspective. Https://ssrn.com/abstract=3101981. Accessed 2 Dec 2018.

Thompson, D. (2017). What On Earth is going on with Bitcoin? The Atlantic. https://www.theatlantic.com/business/archive/2017/11/ bitcoindelusion-conquerworld/547187/. Accessed 30 Nov2017.

Turki, A., K. (1988). Al-Siayasa al-Naqdiyya wal masrifiyya. Muhassah al-risalah, Beirut.

Usmani, M., T. (2010). Islamic or jaded maeeshat o tijarat. Idaratul-Ma'Arif

Wood, G. (2014). Ethereum: A secure decentralized generalised transaction ledger. Ethereum project yellow paper, 151, 1-32, (2014).

Zaharuddin, A. (2017). Bitcoin/Cryptocurrency and persoalan Hukum. Accessed 26 June 2020. www.zaharuddin.net.

Zubaidi, I., B., \& Adam A. (2017). Developing a Digital Currency from an Islamic Perspective: Case of Blockchain Technology. International Business Research, 10, 79-87, (2017). doi:10.5539/ibr.v10n11p79. 
